ANNALES

POLONICI MATHEMATICI

$85.3(2005)$

\title{
Universal sequences for Zalcman's Lemma and $Q_{m}$-normality
}

\author{
by Shahar Nevo (Ramat-Gan)
}

\begin{abstract}
We prove the existence of sequences $\left\{\varrho_{n}\right\}_{n=1}^{\infty}, \varrho_{n} \rightarrow 0^{+}$, and $\left\{z_{n}\right\}_{n=1}^{\infty}$, $\left|z_{n}\right|=1 / 2$, such that for every $\alpha \in \mathbb{R}$ and for every meromorphic function $G(z)$ on $\mathbb{C}$, there exists a meromorphic function $F(z)=F_{G, \alpha}(z)$ on $\mathbb{C}$ such that $\varrho_{n}^{\alpha} F\left(n z_{n}+n \varrho_{n} \zeta\right)$ converges to $G(\zeta)$ uniformly on compact subsets of $\mathbb{C}$ in the spherical metric. As a result, we construct a family of functions meromorphic on the unit disk that is $Q_{m}$-normal for no $m \geq 1$ and on which an extension of Zalcman's Lemma holds.
\end{abstract}

1. Introduction. First we set some notations and conventions. We denote by $\Delta$ the open unit disk in $\mathbb{C}$. For $z \in \mathbb{C}$ and $r>0, \Delta\left(z_{0}, r\right)=$ $\left\{\left|z-z_{0}\right|<r\right\}, \Delta^{\prime}\left(z_{0}, r\right)=\left\{0<\left|z-z_{0}\right|<r\right\}$ and $\bar{\Delta}\left(z_{0}, r\right)=\left\{\left|z-z_{0}\right| \leq r\right\}$. We write $f_{n} \stackrel{\chi}{\Rightarrow} f$ on $D$ to indicate that the sequence $\left\{f_{n}\right\}$ of meromorphic functions on $D$ converges to $f$ uniformly on compact subsets of $D$ in the spherical metric $\chi$, and $f_{n} \Rightarrow f$ on $D$ if the convergence is in the Euclidean metric. For a function $f$ meromorphic on $\mathbb{C}, \Pi(f)$ is the family $\{f(n z): n \in \mathbb{N}\}$, considered as a family of functions on $\Delta$. If $D$ is a domain and $E \subset D$, then the derived set of $E$ with respect to $D$, denoted by $E_{D}^{(1)}$, is the set of accumulation points of $E$ in $D$. For $k \geq 2$ the derived set of order $k$ of $E$ with respect to $D$ is defined inductively by $E_{D}^{(k)}=\left(E_{D}^{(k-1)}\right)_{D}^{(1)}$. The family $\Pi(f)$ is not normal for a nonconstant $f$ meromorphic on $\mathbb{C}$. Normality properties of $\Pi(f)$ were studied from various angles, as will be explained in what follows.

An important and very useful criterion for normality is the following known lemma of L. Zalcman.

Zalcman's Lemma ([Za]). A family $\mathcal{F}$ of functions meromorphic (analytic) on the unit disk $\Delta$ is not normal if and only if there exist

2000 Mathematics Subject Classification: 30D45, 30E10.

Key words and phrases: $Q_{m}$-normal family, Zalcman's Lemma.

Research supported by the German-Israeli Foundation for Scientific Research and Development, G.I.F. Grant No. I-809-234.6/2003. 
(a) a number $0<r<1$;

(b) points $z_{n},\left|z_{n}\right|<r$;

(c) functions $f_{n} \in \mathcal{F}$; and

(d) numbers $\varrho_{n} \rightarrow 0^{+}$,

such that

$$
f_{n}\left(z_{n}+\varrho_{n} \zeta\right) \stackrel{\chi}{\Rightarrow} g(\zeta) \quad \text { on } \mathbb{C},
$$

where $g$ is a nonconstant meromorphic (entire) function on $\mathbb{C}$. Moreover, $g(\zeta)$ can be taken to satisfy the normalization $g^{\#}(\zeta) \leq g^{\#}(0)=1$ for $\zeta \in \mathbb{C}$.

Here $g^{\#}(\zeta)$ is the spherical derivative,

$$
g^{\#}(\zeta)=\frac{\left|g^{\prime}(\zeta)\right|}{1+|g(\zeta)|^{2}} .
$$

Later X. C. Pang extended this result to a criterion of (non)normality by replacing (1) by

$$
\varrho_{n}^{\alpha} f_{n}\left(z_{n}+\varrho_{n} \zeta\right) \stackrel{\chi}{\Rightarrow} g(\zeta) \quad \text { on } \mathbb{C},
$$

where $\alpha$ is any real number satisfying $-1<\alpha<1$. This generalization is very useful to deal with conditions for normality that involve derivatives (see [Pa1], [Pa2]). The interested reader is referred to [PZ] for a modification of Zalcman's Lemma, dealing with families of functions having only multiple zeros.

In [Ne1], we studied the collection of functions $g$ that are limits in (1) for members of the family $\Pi(f)$, where $f(z)$ is a given nonconstant meromorphic function on $\mathbb{C}$.

We have the following result which will be proved in Section 2 .

Theorem A. There exist sequences $\left\{\varrho_{n}\right\}_{n=1}^{\infty}, \varrho_{n} \rightarrow 0^{+}$, and $\left\{z_{n}\right\}_{n=1}^{\infty}$, $\left|z_{n}\right|=1 / 2$, such that for every $\alpha \in \mathbb{R}$ and for every function $G$ meromorphic on $\mathbb{C}$, there is a meromorphic function $F(z)=F_{G, \alpha}(z)$ on $\mathbb{C}$ such that

$$
\varrho_{n}^{\alpha} F\left(n z_{n}+n \varrho_{n} \zeta\right) \stackrel{\chi}{\Rightarrow} G(\zeta) \quad \text { on } \mathbb{C}
$$

and

$$
\overline{\left\{z_{n}: n \geq 1\right\}}=\{|z|=1 / 2\} .
$$

These sequences may be said to be universal with respect to Zalcman's Lemma (or its extensions) for the families $\Pi(F)$.

$Q_{m}$-normality and the family $\Pi(f)$. Let $m$ be a positive integer. A family $\mathcal{F}$ of functions meromorphic on a domain $D$ is called $Q_{m}$-normal on $D$ if each sequence $S=\left\{f_{n}\right\}$ in $\mathcal{F}$ has a subsequence $S^{\prime}=\left\{f_{n_{k}}\right\}$ such that $f_{n_{k}} \stackrel{\chi}{\Rightarrow} f$ on $D \backslash E$, where $f$ is a function on $D \backslash E$ (which happens to be meromorphic or $f \equiv \infty)$, and $E \subset D$ satisfies $E_{D}^{(m)}=\emptyset$. If $\nu \in \mathbb{N}$, then 
a family $\mathcal{F}$ is called $Q_{m}$-normal of order at most $\nu$ on $D$ if in addition $S^{\prime}$ can always be taken such that $E_{D}^{(m-1)}$ contains at most $\nu$ points.

The theory of $Q_{m}$-normal families was developed by C. T. Chuang [Ch]. In [Ne3] it was shown that for every $m \in \mathbb{N}$ and $\nu=1,2,3, \ldots, \infty$ there exists an entire function $f=f_{m, \nu}$ such that $\Pi(f)$ is $Q_{m}$-normal of exact order $\nu$ (i.e., $Q_{m}$-normal of order $\nu$ but not of order $\mu$ for any $\mu<\nu$ ). In $[\mathrm{Ne} 4]$, it was proved that if there exist $a, b \in \widehat{\mathbb{C}}$ such that $f$ attains $a$ and $b$ finitely often each, and $f$ is not a rational function, then $\Pi(f)$ is $Q_{m}$-normal for no $m \in \mathbb{N}$. In [Ne2] the following extension to Zalcman's Lemma was introduced.

N Lemma. Let $\mathcal{F}$ be a family of meromorphic functions in a domain $D$, and $m \geq 1$. In order that $\mathcal{F}$ not be a $Q_{m}$-normal family in $D$, it is necessary and sufficient that there exist

(a) a sequence $S=\left\{f_{n}\right\}_{n=1}^{\infty}$ of functions of $\mathcal{F}$;

(b) a set $E \subset D$ satisfying $E_{D}^{(m)} \neq \emptyset$, and for each point $z \in E$ :

(c) a sequence $\left\{\omega_{n, z}\right\}_{n=1}^{\infty}$ of points in $D$ such that $\omega_{n, z} \rightarrow z$;

(d) a sequence $\eta_{n, z} \rightarrow 0^{+}$; and

(e) a nonconstant function $g_{z}(\zeta)$ meromorphic on $\mathbb{C}$ such that

(f) $f_{n}\left(\omega_{n, z}+\eta_{n, z} \zeta\right) \stackrel{\chi}{\Rightarrow} g_{z}(\zeta)$ on $\mathbb{C}$.

An analogous extension exists for Pang's modification, where for every $-1<\alpha<1$ we have instead of ( $\mathrm{f}$ )

$\left(\mathrm{f}_{\alpha}\right) \eta_{n, z}^{\alpha} f_{n}\left(\omega_{n, z}+\eta_{n, z} \zeta\right) \stackrel{\chi}{\Rightarrow} g_{z}(\zeta)$ on $\mathbb{C}$.

We shall call this extension the extended $N$ Lemma. The "natural" generalization of the N Lemma is not true in the direction $(\Rightarrow)$ for a family $\mathcal{F}$ which is not $Q_{m}$-normal in $D$ for every $m \in \mathbb{N}$. This means that for such an $\mathcal{F}$, there may not exist $E \subset D$ with $E_{D}^{(m)} \neq \emptyset$ for every $m \geq 1$ and a sequence $S$ of functions of $\mathcal{F}$, satisfying (c)-(f) of the N Lemma. (The direction $(\Leftarrow)$ is true of course in this case.)

However, by the result of Theorem A, we shall construct a family $\mathcal{F}$ which is $Q_{m}$-normal for no $m \geq 1$, but satisfies (a)-(f) of the N Lemma, with uncountable set $E$ in (b). This construction is detailed in Theorem B.

REMARK. In [Ne5], we introduced a transfinite extension of the notion of $Q_{m}$-normality and also obtained a "correct" extension of Zalcman's Lemma (or of the N Lemma) for countable ordinal numbers.

THEOREM B. There exists an entire function $F$ such that $\Pi(F)$ is $Q_{m^{-}}$ normal for no $m \geq 1$, and $\Pi(F)$ satisfies (a)-(f) of the $N$ Lemma with $E=\{|z|=1 / 2\}$ in (b). 
The proof of Theorem B is given in Section 3. We also give there an extension of Theorem B in the spirit of condition $\left(\mathrm{f}_{\alpha}\right)$ in the extended N Lemma, for every $\alpha \in \mathbb{R}$.

\section{Proof of Theorem A}

Definition. Let $B$ be a circle in $\mathbb{C}$, centered at $z_{0}$, and let $L$ be a ray with origin at $z_{1}$, tangent to $B$ at $z_{2}$. We say that $L$ is tangent to $B$ from the right (resp. from the left) if

$$
\arg \frac{z_{0}-z_{1}}{z_{2}-z_{1}}>0 \quad\left(\text { resp. } \arg \frac{z_{0}-z_{1}}{z_{2}-z_{1}}<0\right),
$$

where we take the $\operatorname{argument}-\pi<\arg z \leq \pi$.

We now construct a sequence of closed disks, $\left\{B_{k}\right\}_{k=2}^{\infty}$, together with sequences of tangent rays, $\left\{R_{k}\right\}_{k=2}^{\infty}$ and $\left\{L_{k}\right\}_{k=2}^{\infty}$, all originating at $z=0$. For $k=2$, let $B_{2}=\bar{\Delta}(1, \log 2)$ and let $R_{2}$ (resp. $\left.L_{2}\right)$ be the ray originating at $z=0$ and tangent to $B_{2}$ from the right (resp. left). Suppose we have defined $B_{k}, R_{k}, L_{k}$ for $k \geq 2$. Let $B_{k+1}$ be the disk centered on $\{|z|=(k+1) / 2\}$ with radius $\log (k+1)$ such that $L_{k}$ is tangent to $B_{k+1}$ from the right (i.e., $R_{k+1}=L_{k}$ ). $L_{k+1}$ will be the ray that originates at $z=0$ and is tangent to $B_{k+1}$ from the left. It is easy to verify that $B_{k}, R_{k}$ and $L_{k}$ are all well defined. For each $k \geq 2$ denote by

- $\alpha_{k}$ the angle between $R_{k}$ and $L_{k}$, measured counterclockwise;

- $c_{k}$ the center of $B_{k}, c_{k}=(k / 2) e^{i \theta_{k}}$,

where $\left\{\theta_{k}\right\}_{k=2}^{\infty}$ is defined as follows:

$$
\theta_{2}=0, \quad \theta_{3}=\frac{\alpha_{2}+\alpha_{3}}{2}, \quad \theta_{k}=\frac{\alpha_{2}}{2}+\sum_{j=3}^{k-1} \alpha_{j}+\frac{\alpha_{k}}{2}, \quad k \geq 4,
$$

(or $\theta_{k}=\theta_{k-1}+\left(\alpha_{k-1}+\alpha_{k}\right) / 2, k \geq 3$ ). Moreover, denote by

- $T_{k}$ the arc of the circle $\{|z|=k / 2\}$ which subtends the angle $\alpha_{k}$;

- $\left|T_{k}\right|$ the length of $T_{k}$;

- $V_{k}$ the infinite angular sector between $R_{k}$ and $L_{k}$ with angle $\alpha_{k}$, including $R_{k}$ and $L_{k}$;

- $x_{k}, y_{k}$ the points of tangency of $R_{k}$ and $L_{k}$ to $B_{k}$, respectively.

Geometrical considerations yield

$$
\frac{k}{2}-\left|x_{k}\right|=\frac{k}{2}-\left|y_{k}\right| \underset{k \rightarrow \infty}{\longrightarrow} 0^{+} .
$$

Define

$$
A_{k}:=\operatorname{conv}\left(\{0\} \cup B_{k}\right) \quad(\text { convex hull) }
$$


Note that $B_{k}$ and $B_{k+1}$ are pairwise disjoint as can be deduced from (5) (for large enough $k$ ).

We deduce the relations

$$
\begin{gathered}
\frac{\log k}{k / 2}=\sin \frac{\alpha_{k}}{2}, \\
\frac{\left|T_{k}\right|}{k / 2}=\alpha_{k} .
\end{gathered}
$$

Dividing (7) by (6), we get

$$
\frac{\left|T_{k}\right|}{2 \log k}=\frac{\alpha_{k} / 2}{\sin \left(\alpha_{k} / 2\right)} .
$$

From (6) we see that

$$
\alpha_{k} \searrow 0
$$

and

$$
\sum_{k=2}^{\infty} \alpha_{k}=\infty,
$$

which means that the sequence $\left\{e^{i \theta_{k}}\right\}_{k=2}^{\infty}$ encircles the origin infinitely many times.

We now show the existence of $N \in \mathbb{N}$ such that the disks $\left\{B_{k}: k \geq N\right\}$ are pairwise disjoint. From (5) we get $B_{k} \cap B_{k+1}=\emptyset$ for $k \geq N_{1}$. Let $k \geq N_{1}$ and denote by $j_{k}$ the smallest integer that satisfies $j_{k}>k$ and $L_{j_{k}} \subset V_{k}$. By (4) and (9), $\theta_{j_{k}}<\theta_{k}+2 \pi$; so it is sufficient to prove that

$$
B_{k} \cap B_{j_{k}}=\emptyset
$$

for large enough $k$. By (8), $\left|T_{k}\right| / 2 \log k \searrow 1$ as $k \rightarrow \infty$; so there exists some $\beta>1$ such that $\left|T_{k}\right|<\beta \cdot 2 \log k$ for $k \geq 2$. By (9), we conclude that for some $0<C<1$ we have, for $k \geq 2$,

$$
j_{k}-k \geq \frac{2 \pi C}{\alpha_{k}}=\frac{\pi C k}{\left|T_{k}\right|}>\frac{\pi C k}{2 \beta \log k} .
$$

Set

$$
\mu=\frac{\pi C}{2 \beta} .
$$

In order to prove (11), it is sufficient to show that

$$
\left|c_{j_{k}}-c_{k}\right|>\log j_{k}+\log k .
$$

We distinguish two cases.

CASE 1. Suppose that $j_{k}>2 k$. In this case, for some $N_{2}$, we have

$$
\frac{\log k}{j_{k}}+\frac{\log j_{k}}{j_{k}}<\frac{1}{4}<\frac{1-k / j_{k}}{2}, \quad k \geq N_{2} .
$$


CASE 2. Suppose that $j_{k} \leq 2 k$. Then (12) implies that there exists $N_{3}$ such that for $k \geq N_{3}$,

$$
\frac{j_{k}-k}{2} \geq \frac{\mu k}{2 \log k}>\log 2 k+\log k \geq \log j_{k}+\log k .
$$

From (14) and (15), we deduce (13); it follows that $B_{k}, k \geq N=$ $\max \left\{N_{1}, N_{2}, N_{3}\right\}$, are pairwise disjoint as claimed. Now set $G_{N}=A_{N}$ and for $k \geq N$ put $G_{k+1}=G_{k} \cup A_{k+1}$. Then the closed sets $G_{k}$ satisfy

$$
\begin{gathered}
G_{N} \subset G_{N+1} \subset \cdots, \\
\bigcup_{k=N}^{\infty} G_{k}=\mathbb{C}, \\
\operatorname{dist}\left(G_{k}, B_{k+1}\right)>0, \quad G_{k} \cup B_{k+1} \subset G_{k+1} .
\end{gathered}
$$

Define now, for $n \geq 2$,

$$
z_{n}=\frac{1}{2} e^{i \theta_{n}}, \quad \varrho_{n}=\frac{\sqrt{\log n}}{n},
$$

and let $G$ be a meromorphic function on $\mathbb{C}$. For $n \geq N$, set

$$
h_{n}(z)=\varrho_{n}^{-\alpha} G\left(\frac{z-c_{n}}{\sqrt{\log n}}\right) .
$$

By the Mittag-Leffler Theorem, there exists a function $h(z)$ meromorphic on $\mathbb{C}$ such that the poles of $h$ are exactly $\bigcup_{h=N}^{\infty} E_{n}$, where $E_{n}$ is the set of poles of $h_{n}$ in $B_{n}$, and its singular part at any pole in $B_{n}$ is the singular part of $h_{n}$ at that pole. Then for every $n \geq N, \widetilde{h}_{n}=h_{n}-h$ is holomorphic in $B_{n}$.

We define a sequence $\left\{p_{n}\right\}_{n=N}^{\infty}$ of approximating polynomials as follows. We choose $p_{N}$ to satisfy

$$
\max _{z \in B_{N}}\left|p_{N}(z)-\widetilde{h}_{N}(z)\right|<1 / 2^{N} .
$$

The existence of $p_{N}$ is ensured by Runge's Theorem ([Ga, pp. 94-96, Corollary 2 to Runge's Theorem]). Assume that we have defined $p_{N}, p_{N+1}, \ldots, p_{n}$. Again by (18) and Runge's Theorem, there exists a polynomial $p_{n+1}$ such that

$$
\max _{z \in B_{n+1}}\left|p_{n+1}(z)-\widetilde{h}_{n+1}(z)\right|<1 / 2^{n+1}
$$

and

$$
\max _{z \in G_{n}}\left|p_{n+1}(z)-p_{n}(z)\right|<1 / 2^{n+1} .
$$

By (16) and (23), $\left\{p_{n}\right\}$ is a uniform Cauchy sequence on each $G_{n}, n \geq N$, and hence uniformly convergent on $G_{n}$ for $n \geq N$. Thus, by (17), there exists an entire function $p(z)$ such that $p_{n} \Rightarrow p$ on $\mathbb{C}$. Now (20) and (22) 
imply that $p$ is nonconstant. By (23), for $n \geq N$ and $z \in G_{n}$ we have

$$
\left|p_{n}(z)-p(z)\right|<1 / 2^{n} \text {. }
$$

Set $F=p+h$, and let $K$ be a compact set in $\mathbb{C}$. There exists $N^{*} \geq N$ such that $K \subset \Delta\left(0, \sqrt{\log N^{*}}\right)$. From (19)-(22) and (24) and the equality of the singular parts of $F$ and $h_{n}$ in $B_{n}$, we get for $\zeta \in K$ and $n \geq N^{*}$,

$$
\begin{aligned}
& \left|\varrho_{n}^{\alpha} F\left(n z_{n}+n \varrho_{n} \zeta\right)-G(\zeta)\right| \\
& =\left|\varrho_{n}^{\alpha} F\left(c_{n}+\sqrt{\log n} \zeta\right)-\varrho_{n}^{\alpha} h_{n}\left(c_{n}+\sqrt{\log n} \zeta\right)\right| \\
& =\varrho_{n}^{\alpha}\left|p\left(c_{n}+\sqrt{\log n} \zeta\right)-\widetilde{h}_{n}\left(c_{n}+\sqrt{\log n} \zeta\right)\right| \\
& \leq \varrho_{n}^{\alpha}\left|p\left(c_{n}+\sqrt{\log n} \zeta\right)-p_{n}\left(c_{n}+\sqrt{\log n} \zeta\right)\right| \\
& \quad+\varrho_{n}^{\alpha}\left|p_{n}\left(c_{n}+\sqrt{\log n} \zeta\right)-\widetilde{h}_{n}\left(c_{n}+\sqrt{\log n} \zeta\right)\right|<\frac{\varrho_{n}^{\alpha}}{2^{n-1}} \underset{n \rightarrow \infty}{\longrightarrow} 0,
\end{aligned}
$$

and (2) follows. The assertion in (3) can be deduced from (4) and (10). The proof of Theorem A is complete.

3. Proof of Theorem B. Given a nonconstant entire function $G$, let $F$ be an entire function corresponding to $G$ by Theorem A with $\alpha=0$. For $k \geq 3$, set

$$
F_{k}(z)=F(k z) .
$$

Define a sequence $\left\{k_{n}\right\}_{n=1}^{\infty}$ of natural numbers inductively. Set $k_{1}=2$. Suppose we have chosen $k_{n}$. Choose $k_{n+1}$ so that $k_{n+1}>k_{n}$ and $\left|\theta_{k_{n+1}}-2 \pi n\right|$ is minimal. By (4) and (9), we then have $\left|\theta_{k_{n+1}}-2 \pi n\right| \rightarrow 0$ as $n \rightarrow \infty$, so $z_{k_{n}} \rightarrow 1 / 2\left(k_{n+1}\right.$ is chosen such that $z_{k_{n+1}}$ is the $z_{k}$ closest to $z=1 / 2$ at the end of the $n$th lap around the origin by the sequence $\left.\left\{z_{k}\right\}_{k=2}^{\infty}\right)$. We are now ready to define the ingredients in (a)-(f) of the $\mathrm{N}$ Lemma. Let

$$
E=\{|z|=1 / 2\}
$$

and let the sequence $S=\left\{f_{n}\right\}$ of functions of $\Pi(F)$ be defined by

$$
f_{n}=F_{k_{n}} \text {. }
$$

Now for $z=1 / 2$, define

$$
k_{n, 1 / 2}=k_{n}, \quad \omega_{n, 1 / 2}=z_{k_{n, 1 / 2}}, \quad \eta_{n, 1 / 2}=\varrho_{k_{n, 1 / 2}} ;
$$

then by (2), we get

$$
f_{n}\left(\omega_{n, 1 / 2}+\eta_{n, 1 / 2} \zeta\right) \Rightarrow G(\zeta)=g_{1 / 2}(\zeta) \quad \text { on } \mathbb{C} .
$$

It remains to find $\left\{\omega_{n, z}\right\},\left\{\varrho_{n, z}\right\}, g_{z}(\zeta)$ for $z \in E \backslash\{1 / 2\}$. Let $z$ be such a point. By (9) and (10) there exists an increasing sequence $\left\{k_{n, z}\right\}_{n=1}^{\infty}$ of positive integers such that for $n \geq 1$,

$$
k_{n} \leq k_{n, z}<k_{n+1},
$$


and

$$
z_{k_{n, z}} \underset{n \rightarrow \infty}{\longrightarrow} z \text {. }
$$

A sequence $\left\{k_{n, z}\right\}_{n=1}^{\infty}$ that satisfies (26) and (27) is of course not unique. Note that the definition of $k_{n, 1 / 2}$ agrees with (26) and (27). We assert that $k_{n, z} / k_{n} \rightarrow 1$ as $n \rightarrow \infty$. In fact, we will show that the convergence is uniform on $E$. For this purpose, it is enough to show that $k_{n} / k_{n+1} \rightarrow 1$ as $n \rightarrow \infty$. Indeed, by (9), $\sum_{k=k_{n}+1}^{k_{n+1}} \alpha_{k} \rightarrow 2 \pi$ as $n \rightarrow \infty$, and combining it with (6) we get, for large enough $n$,

$$
\frac{\left(k_{n+1}-k_{n}\right) 2 \log k_{n+1}}{k_{n+1} / 2}<\left(k_{n+1}-k_{n}\right) 2 \arcsin \left(\frac{\log k_{n+1}}{k_{n+1} / 2}\right)<\sum_{k=k_{n}+1}^{k_{n+1}} \alpha_{k}<3 \pi .
$$

So if $\underline{\lim } k_{n} / k_{n+1}<1$, we get a contradiction. Set

$$
\omega_{n, z}=z_{k_{n, z}} \frac{k_{n, z}}{k_{n}}, \quad \eta_{n, z}=\varrho_{k_{n, z}} \frac{k_{n, z}}{k_{n}} .
$$

By (26) and (27), we have $\eta_{n, z} \rightarrow 0^{+}$and $\omega_{n, z} \rightarrow z$ as $n \rightarrow \infty$. So together with (2), we have

$$
\begin{array}{r}
\left|f_{n}\left(\omega_{n, z}+\eta_{n, z} \zeta\right)-G(\zeta)\right|=\left|F_{k_{n}}\left(z_{k_{n, z}} \frac{k_{n, z}}{k_{n}}+\varrho_{k_{n, z}} \frac{k_{n, z}}{k_{n}} \zeta\right)-G(\zeta)\right| \\
=\left|F\left(z_{k_{n, z}} k_{n, z}+\varrho_{k_{n, z}} k_{n, z} \zeta\right)-G(\zeta)\right| \Rightarrow 0 \text { on } \mathbb{C}
\end{array}
$$

(thus, $g_{z}=G$ for any $z \in E$ ).

From $(25)$ and (28), it follows that the family $\Pi(F)$ satisfies conditions (a)-(f) of the N Lemma with $E=\{|z|=1 / 2\}$.

We shall now give an extension of Theorem B corresponding to the extension of the $\mathrm{N}$ Lemma by condition $\left(\mathrm{f}_{\alpha}\right)$.

Theorem B*. Let $\alpha \in \mathbb{R}$. Then there exists an entire function $F$ such that $\Pi(F)$ is $Q_{m}$-normal for no $m \geq 1$ and satisfies (a)-(e), $\left(\mathrm{f}_{\alpha}\right)$ of the extended $N$ Lemma with $E=\{|z|=1 / 2 \mid\}$ in (b).

We need the following lemma:

Power Lemma. Let $\mathcal{F}$ be a family of meromorphic functions on a domain $D$ and let $l, m$ be positive integers. Then $\mathcal{F}$ is $Q_{m}$-normal on $D$ if and only if $\mathcal{F}_{l}:=\left\{f^{l}: f \in \mathcal{F}\right\}$ is $Q_{m}$-normal on $D$.

The direction $(\Rightarrow)$ comes from the definition of $Q_{m}$-normality. The opposite direction follows by applying (by negation) the N Lemma with (a)-(f).

4. Proof of Theorem B*. We proceed in two steps. The first step is to find an entire function $F$ that satisfies $(\mathrm{a})-(\mathrm{e}),\left(\mathrm{f}_{\alpha}\right)$ of the extended 
$\mathrm{N}$ Lemma. The second step is to show that $\Pi(F)$ is $Q_{m}$-normal for no $m \geq 1$. For the first step, take any non-constant entire function $G$, and let $F=F_{G, \alpha}$ be the corresponding entire function from Theorem A. We apply the proof of Theorem B with a few modifications. We have to replace (25) by

$$
\eta_{n, 1 / 2}^{\alpha} f_{n}\left(\omega_{n, 1 / 2}+\eta_{n, 1 / 2} \zeta\right) \Rightarrow G(\zeta)=g_{1 / 2}(\zeta) \quad \text { on } \mathbb{C},
$$

and also replace $(28)$ with

$$
\begin{aligned}
\left|\eta_{n, z}^{\alpha} f_{n}\left(\omega_{n, z}+\eta_{n, z} \zeta\right)-G(\zeta)\right| & \\
= & \left|\eta_{n, z}^{\alpha} F_{k_{n}}\left(z_{k_{n, z}} \frac{k_{n, z}}{k_{n}}+\varrho_{k_{n, z}} \frac{k_{n, z}}{k_{n}} \zeta\right)-G(\zeta)\right| \\
& =\left|\eta_{n, z}^{\alpha} F\left(z_{k_{n, z}} k_{n, z}+\varrho_{k_{n, z}} k_{n, z} \zeta\right)-G(\zeta)\right| \Rightarrow 0 \quad \text { on } \mathbb{C} \text {. }
\end{aligned}
$$

The last convergence (to 0 ) is true since $\eta_{n, z} / \varrho_{k_{n, z}} \rightarrow 1$ as $n \rightarrow \infty$.

Now for $-1<\alpha<1$ the non- $Q_{m}$-normality of $\Pi(F)$ for every $m \geq 1$ is ensured by the opposite direction of the extended N Lemma with (a)-(e), $\left(\mathrm{f}_{\alpha}\right)$ and step 2 is done.

For $\alpha \geq 1$ or $\alpha \leq-1$, take $l$ large enough such that $-1<\alpha / l<1$. Then by the previous discussion, there is an entire function $F$ for which $\Pi(F)$ is $Q_{m}$-normal for no $m \geq 1$ and satisfies $(\mathrm{a})-(\mathrm{e}),\left(\mathrm{f}_{\alpha / l}\right)$ of the extended $\mathrm{N}$ Lemma. The family $\Pi\left(F^{l}\right)$ satisfies (a)-(e), $\left(\mathrm{f}_{\alpha}\right)$ of the extended $\mathrm{N}$ Lemma and since $\Pi\left(F^{l}\right)=\Pi(F)_{l}$ it follows by the Power Lemma that $\Pi\left(F^{l}\right)$ is also $Q_{m}$-normal for no $m \geq 1$, as desired. The proof of Theorem B* is complete.

\section{References}

[Ch] C. T. Chuang, Normal Families of Meromorphic Functions, World Sci., 1993.

[Ga] D. Gaier, Lectures on Complex Approximation, Birkhäuser, 1987.

[Ne1] S. Nevo, Normal families of meromorphic functions, Master's thesis, Bar-Ilan Univ., 1995 (in Hebrew).

[Ne2] - Applications of Zalcman's Lemma to $Q_{m}$-normal families, Analysis 21 (2001), 289-325.

[Ne3] - Normality properties of the family $\{f(n z): n \in \mathbb{N}\}$, Comput. Methods Funct. Theory 1 (2001), 375-386.

[Ne4] - Generating quasinormal families of arbitrary degree and order, Analysis 23 (2003), 125-149.

[Ne5] -, Transfinite extension to $Q_{m}$-normality theory, Results Math. 44 (2003), $141-156$.

[Pa1] X. C. Pang, Bloch's principle and normal criterion, Sci. China Ser. A 32 (1989), $782-791$.

[Pa2] -, On normal criterion of meromorphic functions, ibid. 33 (1990), 521-527.

[PZ] X. C. Pang and L. Zalcman, Normal families and shared values, Bull. London Math. Soc. 32 (2000), 325-331. 
[Za] L. Zalcman, A heuristic principle in complex function theory, Amer. Math. Monthly 82 (1975), 813-817.

Department of Mathematics

Bar-Ilan University

52900 Ramat-Gan, Israel

E-mail: nevosh@macs.biu.ac.il

Reçu par la Rédaction le 14.3.2005

Révisé le 19.4.2005 